(C) 2017 IEEE. Personal use of this material is permitted. Permission from IEEE must be obtained for all other uses, in any current or future media, including reprinting/republishing this material for advertising or promotional purposes, creating new collective works, for resale or redistribution to servers or lists, or reuse of any copyrighted component of this work in other works.

Kara, Peter, Cserkaszky, Aron, Barsi, Attila, Papp, Tamas, Martini, Maria and Bokor, László (2017) The interdependence of spatial and angular resolution in the quality of experience of light field visualization. In 2017 International Conference on 3D Immersion (IC3D) Proceedings. Piscataway, U.S. : Institute of Electrical and Electronics Engineers, Inc. ISSN: 2379-1780. ISBN: 9781538646557.

https://doi.org/10.1109/IC3D.2017.8251902 


\title{
THE INTERDEPENDENCE OF SPATIAL AND ANGULAR RESOLUTION IN THE QUALITY OF EXPERIENCE OF LIGHT FIELD VISUALIZATION
}

\author{
Peter A. Kara ${ }^{1,3}$, Aron Cserkaszky², Attila Barsi ${ }^{2}$, Tamas Papp ${ }^{2}$, Maria G. Martini ${ }^{1}$, László Bokor ${ }^{3}$ \\ ${ }^{1}$ WMN Research Group, Kingston University, London, UK \\ \{p.kara, m.martini\}@kingston.ac.uk \\ ${ }^{2}$ Holografika, Budapest, Hungary \\ \{a.cserkaszky, a.barsi, t.papp\}@holografika.com \\ ${ }^{3}$ Budapest University of Technology and Economics, Budapest, Hungary \\ $\{$ kara, bokorl\}@ hit.bme.hu
}

\begin{abstract}
Light field displays provide a natural sense of 3D visual experience through the glasses-free visualization of the content. It is enabled by the smoothness of the horizontal motion parallax, which is determined by the density of source images allocated to a given field of view. This measure is commonly known as angular resolution, and similarly to spatial resolution, has a fundamental effect on the visual experience. In this paper, we investigate how the reduction of angular and spatial resolution affect each other. Our hypothesis is that lowering spatial resolution to a certain extent does not degrade the perception of the parallax effect, in fact, it may improve it. We carried out a series of subjective tests on a real light field display to test this hypothesis, results of which are introduced in this paper.
\end{abstract}

Index Terms - Quality of Experience, light field display, light field visualization, horizontal motion parallax, spatial resolution, image quality.

\section{INTRODUCTION}

At the time of this paper, light field visualization is feasible, commercially available in forms of capture and display utilities, and it is already used in the industry. The continuous and immense research efforts in the area bring closer the day when this technology achieves and enters the level of mainstream multimedia consumption. Until then, much is left to be done regarding development and optimization, and much is left to be understood regarding the Quality of Experience (QoE) of light field displays. Naturally, QoE will be a deciding factor when it comes to the acceptance of this visualization technology from the perspective of the users. Services - such as multimedia streaming - designed for light field displays must take into consideration the capabilities of the Human Visual System (HVS) and the degrees of subjective tolerance should there be any degradation of quality.
The quality of a source content visualized on a light field display can be described by several measures. Source content refers to the state of the content before the display-specific conversion occurs. Although pixels cannot be defined in the visualized light field, as the light rays hit irregular positions (e.g., on the holographic screen, if the architecture employs a holographic diffuser), the source content can be characterized by spatial resolution, which is the image resolution of a single discrete view. The visualized light field can be perceived in a given angle measured from the direction of the screen, known as the field of view (FOV). The source views are converted for the given FOV of the display. It is important to note that after conversion, there are no discrete views at all, as the strength of light field displays originates from the angle-continuous visuals. Currently such displays only provide this continuity horizontally, and they are known as horizontal-only parallax (HOP) displays. In the future, if vertical parallax is supported as well, those will be full-parallax displays. The density of the source for the given FOV determines the smoothness of the horizontal motion parallax; the more views are provided to the converter, the smoother the view transition is when the observer changes position. The number of source views and the FOV together define the angular resolution, as their ratio. In case of a light field display with a 180-degree FOV [1], if the number of source views are 360 , then it means that the angular resolution is either 0.5 degree, or 2 views per degree; both notations are correct.

In case the angular resolution of the source content is insufficiently low, the smoothness of the horizontal motion parallax suffers degradations. Such visual degradations include the crosstalk effect, the appearance of discrete image borders and sudden jumps between the views. Indeed, a very low angular resolution can actually result in having separate discrete views visualized. Approached from an angle of physics and optics, even in that case, the optical engines of the light field display do not project discrete images, but the insufficient input to the converter results in the perception of such. 
Again, the concept of pixels does not apply to light field visualization. This means that if the spatial resolution of the source content is reduced, there is no pixelation that is uniform across the entire scene in the plane of the display. Instead, the visualized content suffers blur. Depending on the content and the degree of resolution reduction, such blur can go unnoticed, or degrade the QoE to a specific level [2].

Even though blur may degrade the user experience, in the world of visualization, it is not always looked at as the enemy. In conventional 2D visualization, computer games consume extra processing power in order to introduce blur and thus make the visuals more realistic. For instance, anti-aliasing is used to eliminate jagged edges (jaggies) by softly blurring them, and the depth of field method simulates the effective focus range by blurring the part of the content that should be out of focus.

In case of light field visualization, an extent of blur can be of help as well. By having a lower spatial resolution and thus introducing blur, it may soften the discrete image borders and the degradations in general that are evoked by having an insufficiently low angular resolution. Our hypothesis is that reducing the spatial resolution for visualization with disturbed horizontal motion parallax will not have a negative effect on the parallax effect, and in fact, it may even improve it. In this paper, we address this research question via a series of subjective tests, carried out on a light field display.

The remainder of this paper is structured as follows: Section 2 provides a brief overview of the relevant related work in light field visualization. Section 3 details the experimental setup of the subjective tests, followed by their results introduced in Section 4. The paper is concluded in Section 5.

\section{RELATED WORK}

Light field first appeared in the scientific literature in 1908, introduced in the publication of integral imaging [3]. The technology can be separated into acquisition (capture light field) and visualization (display light field). In this paper, we solely focus on the quality of the latter.

The methods of visualization quality measurements for light field displays (e.g., spatial resolution, angular resolution etc.) are defined by the International Display Measurement Standard [4]. Such measurements were carried out on light field displays by Kovacs et al. [5] [6]. As no discrete views are displayed on such displays, the angular resolution of a light field display is "determined by the minimal angle of change that rays can reproduce with respect to a single point on the screen" [5].

The content for a light field display can be a series of 2D images, before conversion of course. This can be captured by a single pinhole camera (which either moves on a given arc around the scene, or stays in a static position and viewing angle while the scene itself rotates), an array of cameras or even by virtual cameras (rendered content). The number of views in such image set directly affects the angular resolution of the content, which is one of the leading parameters regarding perceived visual quality. In prior works of Kara et al. [7] [8], the relationship between content angular resolution and the subjective overall quality of light field visualization was studied. The findings indicate that angular resolution reduced to 1 view per degree or lower can severely degrade the user experience.

In order to compensate the loss in view density, intermediate views can be interpolated in order to increase the angular resolution and support the smoothness of the horizontal motion parallax [9]. However, interpolation can only estimate the visual content of the intermediate views, and thus inaccuracies of estimation may result in the degradation of the image quality.

The way angular resolution reduction is perceived is also affected by the observers as well. More precisely, considering only static observers may lessen the sensitivity towards the disturbances in the parallax effect [10]. Such use case scenario, in which an individual is limited to a fixed position and a given viewing angle, is viable in real life, e.g., a light field cinema.

Spatial resolution is also a determining factor of perceived quality, but as it has been explained in Section 1, in case of light field displays, its reduction leads to blur instead of pixelation. Subjective tests carried out with varying source content spatial resolution conclude that even when comparing the extremes of this quality parameter, the experienced difference can still be tolerated [2].

Generally, researches with subjective tests on the visualization quality of light field displays are continuously emerging, together with the core technological advances of this field. Tamboli et al. [11] introduced a novel objective quality assessment metric that take the angular component of visualization into consideration as well. Adhikarla et al. [12] [13] assessed the efficiency of a live capture system and a 3D interface controlled by hand gestures. Dricot et al. [14] conducted a comparison of coding configurations for a light field video service. Viola et al. [15] [16] [17] [18] addressed light field coding and compression, investigated passive and interactive quality evaluations, and introduced new assessment methodologies. The standardized methods of QoE assessment are also investigated by Darukumalli et al. [19]. Kara et al. [20] compared the usability of the FOV values of light field displays and involved Willingness-to-Pay (WTP) in the research. Paudyal et al. [21] highlighted the importance of content selection for light field quality assessment.

The main motivation introduced in this paper is the dynamic adaptive streaming of light field content [22]. Similarly to the solution of dynamic adaptive streaming over HTTP [23] [24] [25], such transmission of light field data would utilize varying quality parameters in order to support playback fluency and reduce the frequency and duration of stalling events, in order to enhance user experience. 
Quality parameters in this case are spatial and angular resolution. The quality switching concept only applies if the transmitted data is not converted to the specifications of the light field display. Converted light field multimedia data has a fixed size based on the duration, regardless of the quality parameters of the source. This means that a given content with significant levels of blur due to low spatial resolution and completely disturbed horizontal motion parallax due to low angular resolution has the exact same converted size as one with exceptionally high spatial and angular resolution.

However, spatial and angular resolution may affect each other, from the aspect of perceived quality. Such attribute can be used in the favor of transmission, if sending less data over the network does not degrade the overall QoE. From the possible interdependencies, in this paper, we investigate how the reduction of spatial resolution affects the perception of angular resolution. As explained earlier, a certain amount of blur caused by lowering the spatial resolution can theoretically lessen the quality disturbances originating from a low angular resolution. The experiment we designed is introduced in details in the following section.

\section{EXPERIMENTAL SETUP}

\subsection{Test Environment and Light Field Display}

The subjective tests were carried out in an isolated laboratory environment. The room allocated for the tests had lighting conditions of approximately $20 \mathrm{~lx}$. The light field display used for visualization during the tests was Holografika's HoloVizio C80 light field cinema system [26] [27] [28]. The holographic screen of the light field display was 3 meters wide and had a brightness of $1500 \mathrm{~cd} / \mathrm{m}^{2}$. The test participants observed the visualized content 4.6 meters away from the screen, which means that the viewing distance was $2.5 \mathrm{H}$, as the height of the screen was 1.85 meters. The default position of viewing was in line with the center of the screen, but test participants had to move a meter to the left and to the right during the given test cases, in order to properly perceive the smoothness of the continuous horizontal motion parallax.

\subsection{Source Visual Stimuli}

We rendered a total of 8 different source visual stimuli for the research (see Figure 1). The stimuli can be clustered into 4 categories, 2 stimuli each: stimulus A and B were collections of simple shapes, C and D were mathematical bodies with high structural complexities, $\mathrm{E}$ and $\mathrm{F}$ were spatially diverse and textured objects, and $\mathrm{G}$ and $\mathrm{H}$ were laser-scanned real statues. The depth values of the source stimuli were crucial during selection, as depth plays a major role in the perception of reduced angular resolution [9] [10].

$\begin{array}{lll}\text { Condition number } & \begin{array}{l}\text { Spatial resolution } \\ 1440 \times 1080\end{array} & \begin{array}{l}\text { Number of views } \\ 1\end{array} \\ 2 & 1440 \times 1080 & 45 \\ 3 & 1440 \times 1080 & 30 \\ 4 & 1024 \times 768 & 135 \\ 5 & 1024 \times 768 & 45 \\ 6 & 1024 \times 768 & 30 \\ 7 & 640 \times 480 & 135 \\ 8 & 640 \times 480 & 45 \\ 9 & 640 \times 480 & 30\end{array}$

Table 1: Investigated test conditions.

\subsection{Test Stimuli}

The test stimuli were directly rendered in the given spatial and angular resolution values, so no post-rendering transformation of any sort was necessary. We selected 3 values for each, and rendered the stimuli in all 9 combinations (see Table 1). The choice of spatial [2] and angular [7] [8] values was based on the findings of prior researches.

The light field display was calibrated to enable a 45-degree field of view. This means that if a visual stimulus had 45 views, then it had an angular resolution of 1 view per degree.

\subsection{Test Protocol}

The subjective test was designed as a paired comparison, focusing on the variation of spatial resolution at given degrees of angular resolution. The test participants had to rate the smoothness of the horizontal motion parallax; the evaluation focused on the angular resolution. A seven-point comparison scale (Much Worse, Worse, Slightly worse, Same, Slightly better, Better, Much better) was chosen, based on ITU-R Rec. BT-500.13 [29]. A Degradation Category Rating (DCR) scale [30] would have been a viable choice for the test design, if the focus of the research had been the overall visual quality. However, using such a scale would have eliminated the possibility of assessing improvement in the perceived quality.

The investigated test conditions were paired in a way that only spatial resolution varied. As stated earlier, there were 3 conditions with a given angular resolution, defined by the number of views. If we take the highest number of source views (135), and pair the conditions with each other, we get 3 comparisons ( 1 with 4, 4 with 7 and 1 with 7). As there were 3 different angular resolution values, the total number of comparisons was 9 .

Each series of comparisons ( 9 in total) was run on each source stimulus ( 8 in total), so the sum of comparisons was 72. The comparison stimuli and also the comparisons themselves were separated by 5 -second blank screens, and each stimuli (144 in total) was displayed for 10 seconds. The total duration of each subjective test was approximately 45 minutes, with the training phase included. 


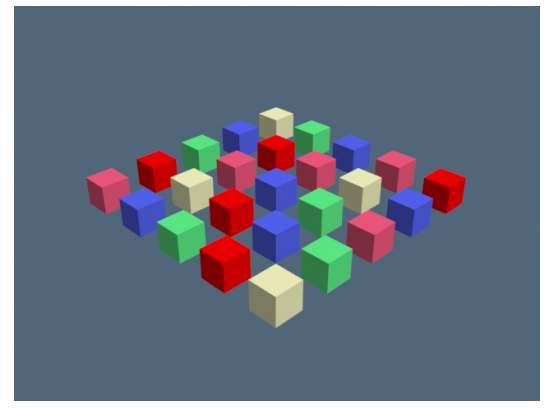

(a) Stimulus A

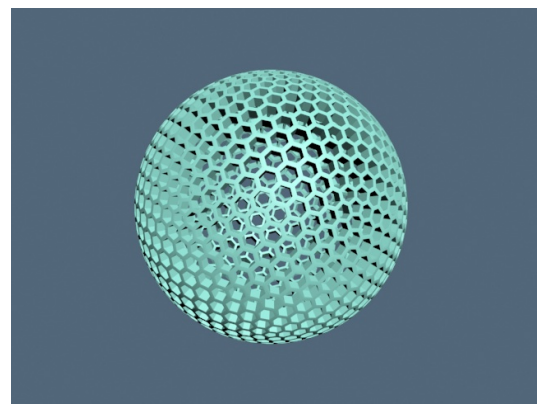

(c) Stimulus C

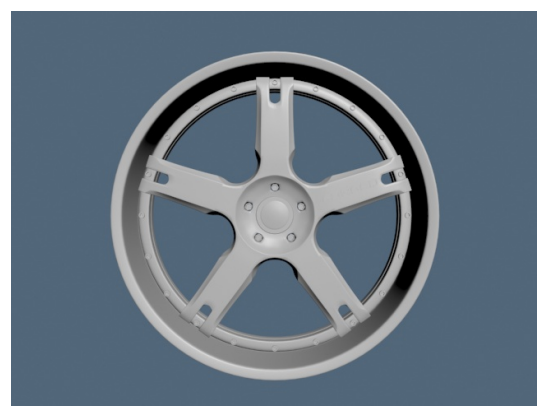

(e) Stimulus E

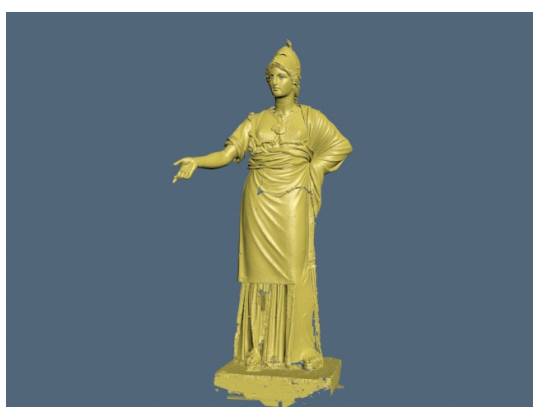

(g) Stimulus G

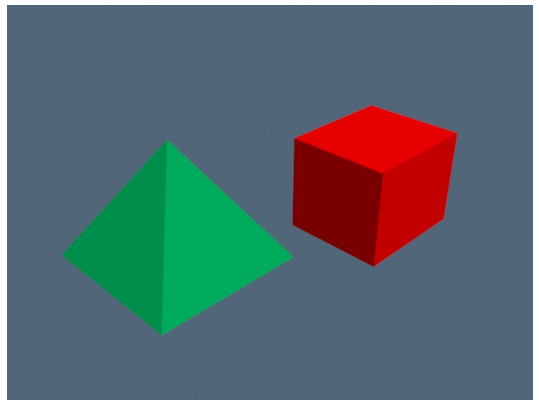

(b) Stimulus B

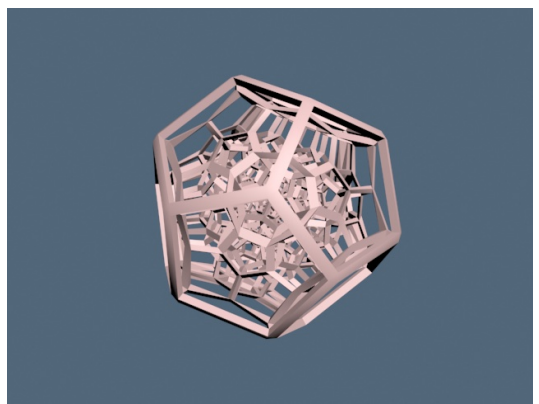

(d) Stimulus D

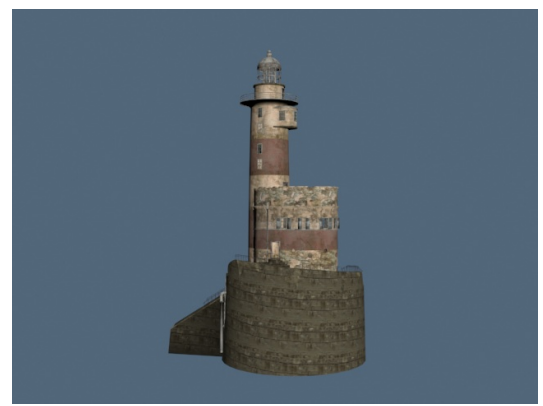

(f) Stimulus F

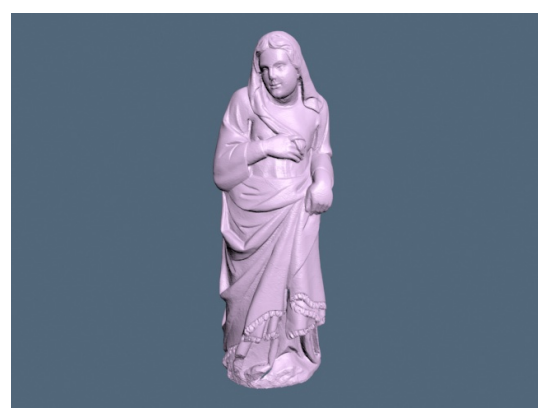

(h) Stimulus H

Fig. 1: The source stimuli of the research. 


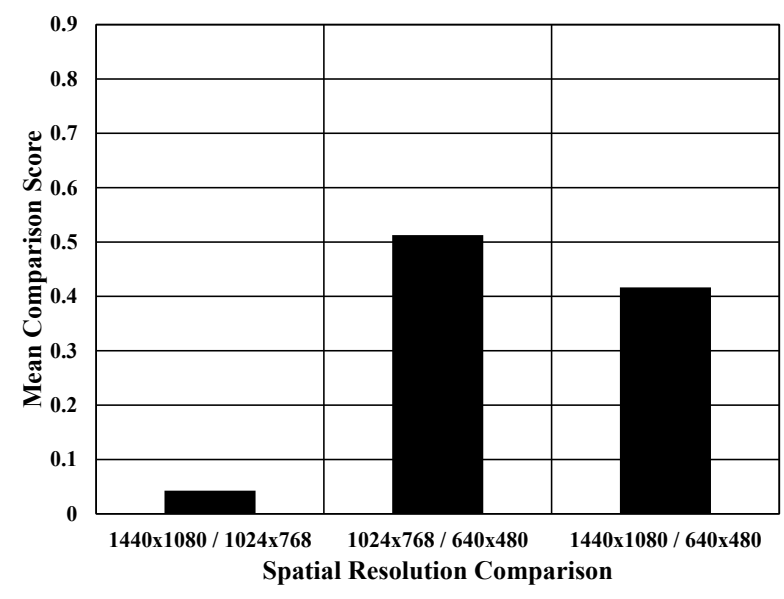

Fig. 2: Average comparison scores for 135 source views.

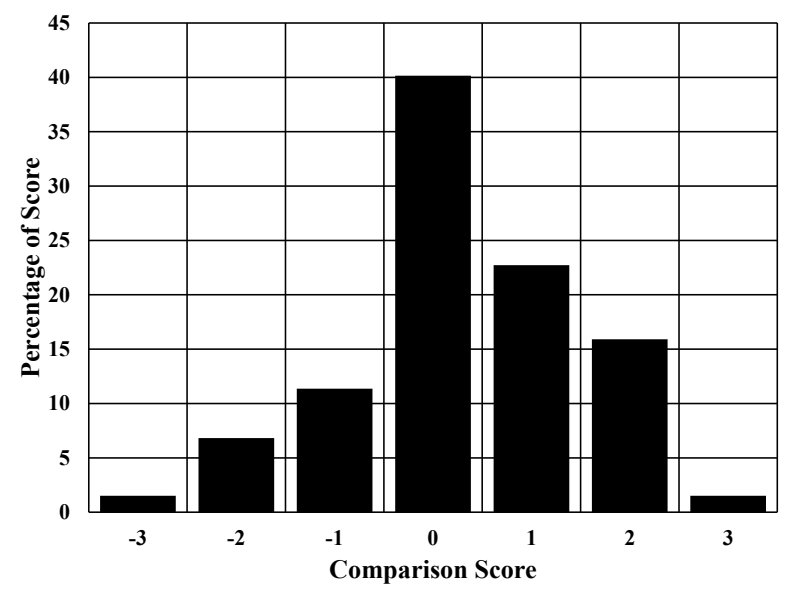

Fig. 3: Scoring distribution for 135 source views.

\subsection{Test Participants}

A total of 22 test participants completed the subjective tests. The average age was 31 , and test participants were selected from an age interval ranging from 18 to 58 . From the 22 participants 16 were male and 6 were female.

\section{RESULTS}

As the comparisons defined by the test protocol only include transitions between given spatial resolutions, we cluster the obtained results by the different angular resolutions of the stimuli, given in the number of source views.

\subsection{5 source views}

The obtained mean comparison scores for 135 source views (see Figure 2) show that the difference in the perceived smoothness of the continuous horizontal motion parallax when degrading from $1440 \times 1080$ to $1024 \times 768$ source spatial reso-

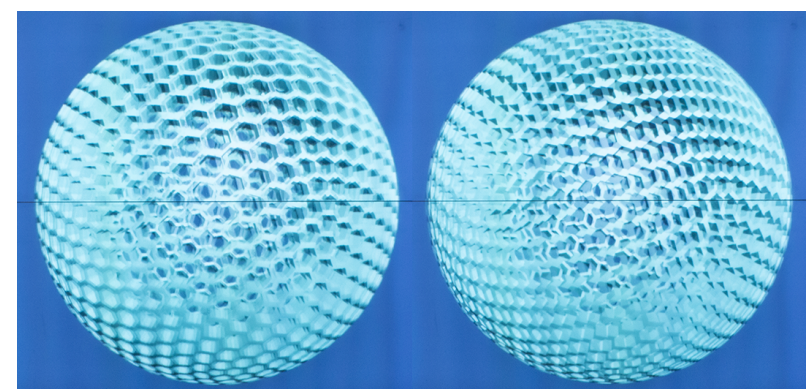

Fig. 4: Stimulus C converted from 45 (left) and 30 (right) source views, as seen by a pinhole camera.

lution is negligible. Even though a low number of test participants experienced a slight improvement regarding 3D continuity, a very similar number and extent of comparative ratings were present in the opposite scoring direction as well.

For the other two transitions between spatial resolution at this degree of angular resolution, the mean scores indicate a statistically equivalent increase. At this point, it needs to be noted that this given angular resolution (3 source views per degree) already provided an excellent level of parallax smoothness and further increase on this light field display would not have resulted in perceivable visual benefits [7]. Yet the scores show positive comparisons for these transitions, but not for the one between the highest resolutions.

We anticipated that the transition from $1440 \times 1080$ to $1024 \times 768$ source spatial resolution would receive the smallest gains, especially with high angular resolution, as such degradation is not evident to be perceived. In a prior research [2], a similar transition of spatial resolution was not detected by approximately third of the test participants.

In general, the gain at this high angular resolution is not significant, as all statistical analyses concluded insignificance. This is also reflected in the scoring distribution (see Figure 3), which is centered on zero difference. It is a notable fact that more than $40 \%$ of the test participants did not experience a difference during the 3 comparisons at 135 source views.

\subsection{5 source views}

With 45 rendered source views before conversion, the content angular resolution was 1 view per degree. It is arguable whether such angular resolution is sufficient for light field visualization or not. It heavily depends on the content, the display and the viewing conditions. Certain contents with great depth values, detailed structures and surfaces may be critically sensitive to angular resolution reduction [9], resulting in the severe degradation of user experience (see Figure 4). While the impact of the light field display itself is rather evident, viewing conditions are less investigated, yet they may affect the perception of the smoothness of the continuous horizontal motion parallax [10]. 


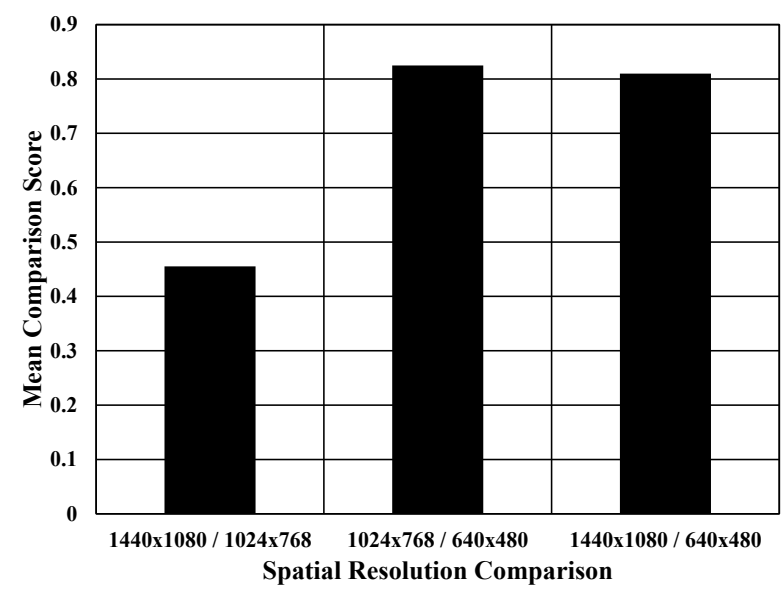

Fig. 5: Average comparison scores for 45 source views.

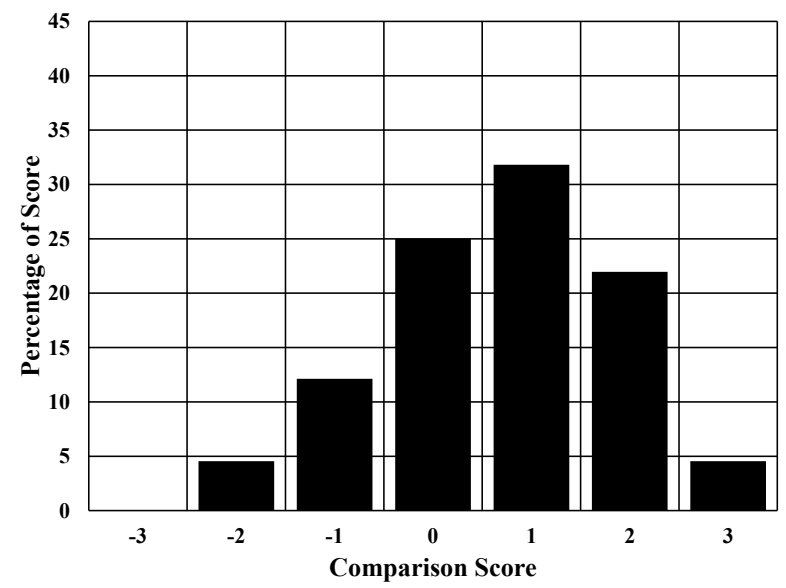

Fig. 6: Scoring distribution for 45 source views.

The mean results (see Figure 5) indicate higher comparison scores for the transition from $1440 \times 1080$ to $1024 \times 768$ source spatial resolution for this given angular resolution. The other transitions also received better ratings in the aspect of perceived angular resolution, and these managed to reach the level of significant visual benefits.

The distribution of scores (see Figure 6) reveals that for all 3 transitions, the comparison score Slightly better (32\%) was used the most frequently to describe the change in the smoothness of horizontal parallax, followed by Same $(25 \%)$ and Better (22\%), making these the dominant ratings at this degree of angular resolution. Practically, nearly $60 \%$ of the scores of the test participants reflected a level of perceptual improvement regarding the parallax effect.

We conclude that reducing the spatial resolution of the source content at this angular resolution can have a positive effect on the perceived parallax effect, as the spatial resolution degradation lessens the observability of the investigated visual phenomena (e.g., crosstalk effect).

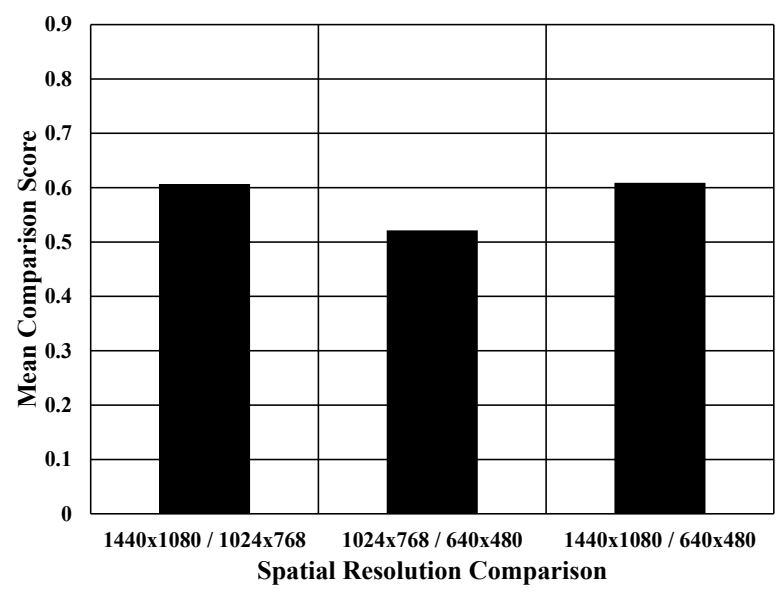

Fig. 7: Average comparison scores for 30 source views.

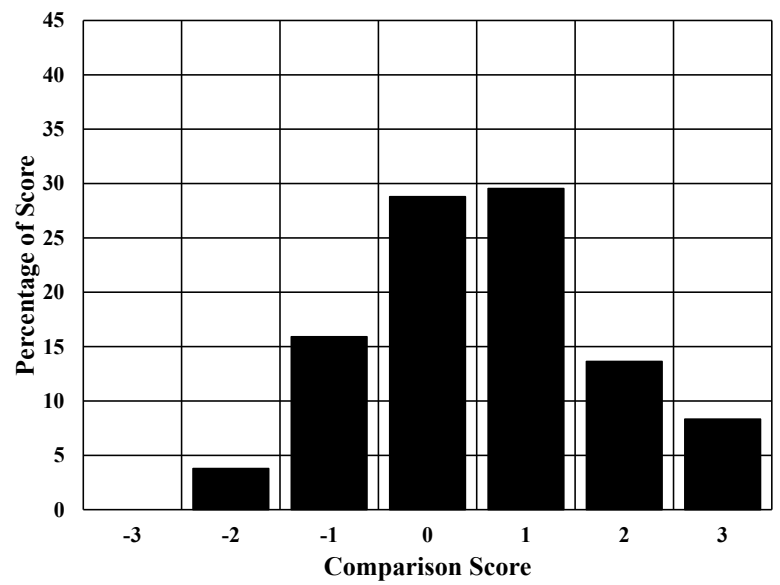

Fig. 8: Scoring distribution for 30 source views.

\subsection{0 source views}

For the comparison of the highest spatial resolutions, the scores obtained at 30 source views (see Figure 7) show further increase compared to the previously detailed cases of angular resolution, and scoring is statistically indifferent (one-way ANOVA, post-hoc Tukey HSD test, Bonferroni comparison) for all 3 comparisons. Yet these means are lower than initially expected.

The results suggest that the visual benefit at hand is not a clear one-way function; one could think that the lower the angular resolution is, the more spatial resolution reduction can improve its perception. Theoretically yes, that would be the case, however, such low levels of angular resolution also affect the capability to distinguish changes in general. Stimulus $\mathrm{C}$ of the research can easily portray this (see Figure 4), as the model itself became difficult to recognize when the rendered content was converted and visualized on the light field display. Still, such reduction in source spatial resolution may indeed have its benefits. 
As there is an increase of comparison scores for the first comparison but a decrease for the second and the third, we can see a slight realignment ( 0.5 shift to the left) in the distribution (see Figure 8). Regarding negative scores in general (which mean degradations in the perception of horizontal parallax), they are present at all 3 angular resolutions, and always around the same extent (17-19\%). There were certain test participants who did not find spatial resolution reduction beneficial in the aspect of angular resolution, and found the additional blur bothersome, resulting in negative assessment.

\section{CONCLUSION}

In this paper, we introduced our research results on how the reduction of source content spatial resolution affects the perception of the parallax effect. Our results indicate that such transition can be beneficial, and that the highest amount of improvement can be obtained at an angular resolution of 1 source view per degree. We found that switching between high spatial resolutions does not make a significant difference at sufficiently high angular resolutions, and that such switching may have similar effects as switching between lower resolutions, if the angular resolution is low. The obtained scores had rating distributions centered on 0 in case of 135 source views (no difference), 1 in case of 45 source views (slight improvement) and 0.5 in case of 30 source views (between the two).

The research only investigated one component of the overall user experience. However, this single component is a critical one in the subjective evaluation of light field visualization. In case the perceived degradations caused by blur of lower angular resolution do not affect QoE more in a negative way than the positive effect on the smoothness of the horizontal motion parallax, the global QoE would not be reduced, yet the required unconverted data would be less. In future works, we aim to use the gathered knowledge on the perceived quality of light field visualization to support new transmission protocols for light field content delivery, especially dynamic adaptive streaming.

\section{ACKNOWLEDGMENTS}

The research in this paper was done as a part of and was funded from the European Unions Horizon 2020 research and innovation program under the Marie Sklodowska-Curie grant agreement No 643072, Network QoE-Net, and No 676401, ETN-FPI. The authors would like to thank Viktoria Major, Zsuzsa Dobranyi, Gabor Puhr and Gaspar Balogh (all affiliated with Holografika) for their contributions to the research and all the participants of the subjective tests.

\section{REFERENCES}

[1] Holografika. Holovizio 80WLT light field display. http://www.holografika.com/Documents/ HoloVizio_80WLT-emailsize.pdf (retrieved June 2017).

[2] Peter A. Kara, Péter T. Kovács, Maria G. Martini, Attila Barsi, Kristóf Lackner, and Tibor Balogh. Viva la Resolution: The Perceivable Differences between Image Resolutions for Light Field Displays. In 5th ISCA/DEGA Workshop on Perceptual Quality of Systems (PQS 2016), Berlin, 2016.

[3] Gabriel Lippmann. Epreuves reversibles. photographies integrals. Comptes-Rendus Academie des Sciences, 146:446-451, 1908.

[4] Society for Information Display. The International Display Measurement Standard v1.03. 2012.

[5] Péter T. Kovács, Atanas Boev, Robert Bregović, and Atanas Gotchev. Quality measurements of 3D lightfield displays. In Proc. Eighth International Workshop on Video Processing and Quality Metrics for Consumer Electronics (VPQM), 2014.

[6] Péter T. Kovács, Kristóf Lackner, Attila Barsi, Ákos Balázs, Atanas Boev, Robert Bregović, and Atanas Gotchev. Measurement of perceived spatial resolution in 3D light-field displays. In International Conference on Image Processing (ICIP), pages 768-772. IEEE, 2014.

[7] Peter A. Kara, Maria G. Martini, Peter T. Kovács, Sandor Imre, Attila Barsi, Kristóf Lackner, and Tibor Balogh. Perceived quality of angular resolution for light field displays and the validity of subjective assessment. In International Conference on $3 D$ Imaging (IC3D), Liege, 2016.

[8] Peter A. Kara, Péter T. Kovács, Suren Vagharshakyan, Maria G. Martini, Attila Barsi, Tibor Balogh, Aleksandra Chuchvara, and Ahmed Chehaibi. The Effect of Light Field Reconstruction and Angular Resolution Reduction on the Quality of Experience. In 12th International Conference on Signal Image Technology \& Internet Based Systems (SITIS) 3rd International Workshop on Quality of Multimedia Services (QUAMUS), Naples, 2016.

[9] Aron Cserkaszky, Peter A. Kara, Attila Barsi, and Maria G. Martini. To Interpolate or not to Interpolate: Subjective Assessment of Interpolation Performance on a Light Field Display. In IEEE International Conference on Multimedia and Expo (ICME) 8th Workshop on Hot Topics in 3D Multimedia (Hot3D), Hong Kong, 2017. 
[10] Peter A. Kara, Aron Cserkaszky, Subbareddy Darukumalli, Attila Barsi, and Maria G. Martini. On the Edge of the Seat: Reduced Angular Resolution of a Light Field Cinema with Fixed Observer Positions. In 9th International Conference on Quality of Multimedia Experience (QoMEX), Erfurt, 2017.

[11] Roopak R. Tamboli, Balasubramanyam Appina, Sumohana Channappayya, and Soumya Jana. Supermultiview content with high angular resolution: 3D quality assessment on horizontal-parallax lightfield display. Signal Processing: Image Communication, 47:42$55,2016$.

[12] Vamsi K. Adhikarla, Fabio Marton, Tibor Balogh, and Enrico Gobbetti. Real-time adaptive content retargeting for live multi-view capture and light field display. The Visual Computer, 31(6-8):1023-1032, 2015.

[13] Vamsi K. Adhikarla, Jaka Sodnik, Peter Szolgay, and Grega Jakus. Exploring direct 3D interaction for full horizontal parallax light field displays using leap motion controller. Sensors, 15(4):8642-8663, 2015.

[14] Antoine Dricot, Joel Jung, Marco Cagnazzo, Béatrice Pesquet, Frédéric Dufaux, Péter T. Kovács, and Vamsi K. Adhikarla. Subjective evaluation of super multi-view compressed contents on high-end light-field 3D displays. Signal Processing: Image Communication, 39:369-385, 2015.

[15] Irene Viola, Martin Rerabek, and Touradj Ebrahimi. Impact of interactivity on the assessment of quality of experience for light field content. In 9th International Conference on Quality of Multimedia Experience (QoMEX), 2017.

[16] Irene Viola, Martin Rerabek, Tim Bruylants, Peter Schelkens, Fernando Pereira, and Touradj Ebrahimi. Objective and subjective evaluation of light field image compression algorithms. In Picture Coding Symposium (PCS), 2016, pages 1-5. IEEE, 2016.

[17] Irene Viola, Martin Rerabek, and Touradj Ebrahimi. A new approach to subjectively assess quality of plenoptic content. In Applications of Digital Image Processing XXXIX, volume 9971, pages 99710X-1. SPIE, 2016.

[18] Irene Viola and Touradj Ebrahimi. A new framework for interactive quality assessment with application to light field coding. In Proceedings of SPIE, number EPFLCONF-230109, 2017.

[19] Subbareddy Darukumalli, Peter A. Kara, Attila Barsi, Maria G. Martini, Tibor Balogh, and Ahmed Chehaibi. Performance comparison of subjective assessment methodologies for light field displays. In Signal Processing and Information Technology (ISSPIT), 2016
IEEE International Symposium on, pages 28-33. IEEE, 2016.

[20] Peter A. Kara, Péter T. Kovács, Maria G. Martini, Attila Barsi, Kristóf Lackner, and Tibor Balogh. From a Different Point of View: How the Field of View of Light Field Displays affects the Willingness to Pay and to Use. In 2016 Eighth International Workshop on Quality of Multimedia Experience (QoMEX), Lisbon, 2016.

[21] Pradip Paudyal, Jesus Gutierrez, Patrick Le Callet, Marco Carli, and Federica Battisti. Characterization and selection of light field content for perceptual assessment. In 9th International Conference on Quality of Multimedia Experience (QoMEX 2017), 2017.

[22] Peter A. Kara, Aron Cserkaszky, Attila Barsi, Maria G. Martini, and Tibor Balogh. Towards Adaptive Light Field Video Streaming. COMSOC MMTC Communications - Frontiers, 2017.

[23] Thomas Stockhammer. Dynamic adaptive streaming over HTTP-: standards and design principles. In Proceedings of the second annual ACM conference on Multimedia systems, pages 133-144. ACM, 2011.

[24] Ognen Ognenoski, Moustafa M. Nasralla, Manzoor Razaak, Maria M. Martini, and Peter Amon. Dash-based video transmission over lte networks. In Communication Workshop (ICCW), 2015 IEEE International Conference on, pages 1783-1787. IEEE, 2015.

[25] Ozgur Oyman and Sarabjot Singh. Quality of experience for HTTP adaptive streaming services. IEEE Communications Magazine, 50(4), 2012.

[26] Tibor Balogh. The holovizio system. In Electronic Imaging 2006, pages 60550U-60550U. International Society for Optics and Photonics, 2006.

[27] Holografika. HoloVizio C80 light field cinema system. www.holografika.com/Documents/HoloVizio_C80.pdf (retrieved June 2017).

[28] Tibor Balogh, Zsolt Nagy, Péter T. Kovács, and Vamsi K. Adhikarla. Natural 3d content on glasses-free light-field $3 \mathrm{~d}$ cinema. In IS\&T/SPIE Electronic Imaging, pages $86480 \mathrm{~F}-86480 \mathrm{~F}$. International Society for Optics and Photonics, 2013.

[29] ITU-R Rec. BT.500-13: Methodology for the subjective assessment of the quality of television pictures. 2012.

[30] ITU-T Rec. P.910: Subjective video quality assessment methods for multimedia applications. 2008. 